Acta Crystallographica Section E

Structure Reports

Online

ISSN 1600-5368

\section{4-Amino-13-(1-naphthyl)-[2,2]paracyclo- phane}

\section{Junshan Sun, ${ }^{\mathrm{a}}$ Yanmin Huo, ${ }^{\mathrm{b}}$ Rentao $\mathrm{Wu},{ }^{\mathrm{c}}$ Jikun $\mathrm{Li}^{\mathrm{a}}$ and Yudao $\mathrm{Ma}^{\mathrm{d} *}$}

aDepartment of Materials and Chemical Engineering, Taishan University, 271021 Taian, Shandong, People's Republic of China, 'bShandong Institute of Supervision \& Inspection of Product Quality, 250100 Jinan, Shandong, People's Republic of China, 'Department of Chemistry, Taishan University, 271021 Taian, Shandong, People's Republic of China, and dDepartment of Chemistry, Shandong University, 250100 Jinan, Shandong, People's Republic of China

Correspondence e-mail: ydma@sdu.edu.cn

Received 5 November 2007; accepted 25 February 2008

Key indicators: single-crystal X-ray study; $T=293 \mathrm{~K}$; mean $\sigma(\mathrm{C}-\mathrm{C})=0.007 \AA$; $R$ factor $=0.054 ; w R$ factor $=0.160 ;$ data-to-parameter ratio $=7.8$.

The title compound [systematic name: $1^{2}$-amino- $4^{2}$-(1-naphthyl)-1,4(1,4)-dibenzenacyclohexaphane], $\mathrm{C}_{26} \mathrm{H}_{23} \mathrm{~N}$, was synthesized from 4-amino-13-bromo-[2,2]paracyclophane and 1naphthaleneboronic acid in the presence of 1,4-dioxane. It is a new cyclophane-derived compound which can be regarded as a prospective ligand for asymmetric synthesis and catalysis. The benzene rings of the paracyclophane units are very slightly deformed from planarity as shallow boats.

\section{Related literature}

For related literature on paracyclophane chemistry, see: Cipiciani et al. (1997); on diphosphanes, see: Pye et al. (1997); on oxazoline-phosphanes, see: Wu et al. (2003); on oxazolineimidazolium, see: Bolm et al. (2003); on oxazoline-selenides, see: Hou et al. (2000); on oxazoline-alcohols, see: Wu et al. (2001).

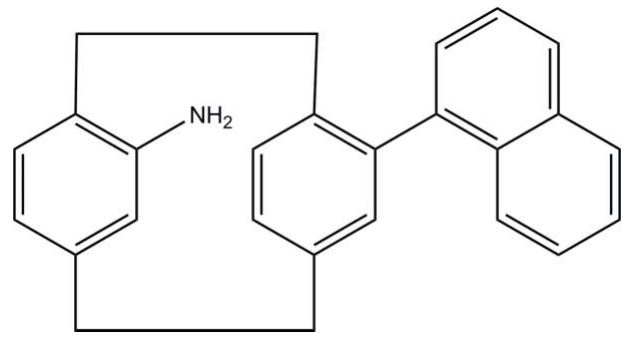

\section{Experimental}

Crystal data

$\mathrm{C}_{26} \mathrm{H}_{23} \mathrm{~N}$

$M_{r}=349.45$

Orthorhombic, $P 2_{1} 2_{1} 2_{1}$

$a=8.5261(5) \AA$

$b=12.8123(8) \AA$

$c=17.2065(11) \AA$

$V=1879.6(2) \AA^{3}$

$Z=4$

Mo $K \alpha$ radiation

$\mu=0.07 \mathrm{~mm}^{-1}$

$T=293(2) \mathrm{K}$

$0.15 \times 0.12 \times 0.10 \mathrm{~mm}$

\section{Data collection}

Bruker SMART APEXII CCD area-detector diffractometer Absorption correction: multi-scan (SADABS; Sheldrick, 1996) $T_{\min }=0.990, T_{\max }=0.993$

9879 measured reflections 1901 independent reflections 1690 reflections with $I>2 \sigma(I)$ $R_{\text {int }}=0.026$

Refinement

$R\left[F^{2}>2 \sigma\left(F^{2}\right)\right]=0.054$

$w R\left(F^{2}\right)=0.159$

$S=1.00$

1901 reflections

245 parameters

$\mathrm{H}$-atom parameters constrained

$\Delta \rho_{\max }=0.37 \mathrm{e} \AA^{-3}$

$\Delta \rho_{\min }=-0.33 \mathrm{e}^{-3}$

Data collection: APEX2 (Siemens, 1996); cell refinement: SAINT (Siemens, 1996); data reduction: $S A I N T$; program(s) used to solve structure: SHELXS97 (Sheldrick, 2008); program(s) used to refine structure: SHELXL97 (Sheldrick, 2008); molecular graphics: SHELXTL (Sheldrick, 2008); software used to prepare material for publication: SHELXTL.

Financial support from the National Natural Science Foundation of China (grant Nos. 20441004, 20671059) and the Department of Science and Technology of Shandong Province is gratefully acknowledged.

Supplementary data and figures for this paper are available from the IUCr electronic archives (Reference: KJ2079).

\section{References}

Bolm, C., Focken, T. \& Raabe, G. (2003). Tetrahedron Asymmetry, 14, 17331746.

Cipiciani, A., Fringuelli, F., Mancini, V., Piermatti, O., Scappini, M. \& Ruzziconi, R. (1997). Tetrahedron, 53, 11853-11858.

Hou, X.-L., Wu, X.-W., Dai, L.-X. \& Cao, B.-X. (2000). Chem. Commun. pp. $1195-1196$.

Pye, P. J., Rossen, K., Reamer, R. A., Tsou, N. N., Volante, R. P. \& Reider, P. J. (1997). J. Am. Chem. Soc. 119, 6207-6208.

Sheldrick, G. M. (1996). SADABS. University of Göttingen, Germany.

Sheldrick, G. M. (2008). Acta Cryst. A64, 112-122.

Siemens (1996). APEX2 and SAINT. Siemens Analytical X-ray Systems Inc., Madison, Wisconsin, USA.

Wu, X.-W., Hou, X.-L., Dai, L.-X., Tao, J., Cao, B.-X. \& Sun, B. J. (2001). Tetrahedron Asymmetry, 12, 529-532.

Wu, X.-W., Yuan, X. K., Sun, W., Zhang, M.-J. \& Hou, X.-L. (2003). Tetrahedron Asymmetry, 14, 107-112. 


\section{supporting information}

Acta Cryst. (2008). E64, o650 [doi:10.1107/S1600536808005333]

\section{4-Amino-13-(1-naphthyl)-[2,2] paracyclophane}

\section{Junshan Sun, Yanmin Huo, Rentao Wu, Jikun Li and Yudao Ma}

\section{S1. Comment}

The chemistry of [2.2]paracyclophanes has attracted the interest of researchers since the middle of the last century. After a standstill period, investigations in this area have received a new impulse (Cipiciani et al., 1997) and recently there has been notable progress especially regarding the synthesis of new derivatives. [2.2]paracyclophane is unique as the strain in the molecule has become so large that the benzene rings have been substantially bent from planarity. The configurationally rigid [2.2]paracyclophanyl unit makes the design of chiral ligands of different types possible. The [2.2]paracyclophane ligand has previously been included in diphosphanes, (Pye et al., 1997) oxazoline-phosphanes,(Wu et al., 2003) oxazoline-imidazolium,(Bolm et al., 2003) oxazoline-selenides, (Hou et al., 2000) oxazoline-alcohols,(Wu et al., 2001) and Schiff base phenols.

The benzene rings in the $[2,2]$ paracyclophane are not planar. Their conformation can be described as an asymmetric boat conformation. The benzene $\mathrm{C}$ atoms which are directly bonded to the ethylene links of the paracyclophane deviate significantly from the least-squares planes running through the other four benzene $\mathrm{C}$ atoms. The largest deviations are found for the atoms C3 $[0.117$ (5) $\AA]$ and C12 [0.146 (4) $\AA]$, which are the atoms closest to the amino and naphtyl substituents of the benzene rings. The angle between the planes through the benzene rings is $6.0(2)^{\circ}$. The $\mathrm{N} 1-\mathrm{C} 1$ bond length lies between the expected values for a $\mathrm{C}-\mathrm{N}$ and a $\mathrm{C}=\mathrm{N}$ bond, which is probably caused by $\mathrm{p}-\pi$ conjugation.

\section{S2. Experimental}

A solution of 4-amino-13-bromo [2,2]paracyclophane (501.3 mg, $1.66 \mathrm{mmol}$ ), 1-naphthaleneboronic acid (428.3 mg, 2.49 mmol), KF (289.7 mg, $4.98 \mathrm{mmol})$, Pd-DPPF (13.6 mg, $0.0166 \mathrm{mmol}$ ) in 1,4-dioxane (5 ml) was stirred at 353-363k for $24 \mathrm{~h}$ under a slight overpressure of nitrogen. After this, reagents were added to the mixture at $24 \mathrm{~h}$ intervals. 1naphthaleneboronic acid (0.55 mmol), KF (72.4 mg, $1.245 \mathrm{mmol})$, Pd-DPPF (13.6 mg, $0.0166 \mathrm{mmol})$ were added to the flask in the first two times, in the last two times, 1-naphthaleneboronic acid ( $0.55 \mathrm{mmol}), \mathrm{KF}$ ( $72.4 \mathrm{mg}, 1.245 \mathrm{mmol}), \mathrm{Pd}$ DPPF (6.78 mg, $0.0083 \mathrm{mmol}$ ) were added. The flask was kept at 353-363 $\mathrm{K}$ and stirred the whole time. After completion of the reaction, as indicated by TLC, water $(5 \mathrm{ml})$ was added and the solution was filtered. The solution was extracted by dichloromethane $(30 \mathrm{ml})$ and the solvent was removed on a rotary evaporator. The solid was subjected to chromatography on silica gel (eluent: petroleum ether / ethyl acetate =20:1). Pure product was isolated (yield 84.6\%). Analysis, calculated for $\mathrm{C}_{26} \mathrm{H}_{23} \mathrm{~N}$ : C, 89.36; H, 6.63; N, 4.01. Found: $\mathrm{C}, 89.03 ; \mathrm{H}, 6.62 ; \mathrm{N}, 3.93$. The elemental analyses were performed with a Perkin Elmer PE2400II.

\section{S3. Refinement}

All the $\mathrm{H}$ atoms could be found in the difference Fourier maps. Nevertheless, they were placed into the idealized positions and refined in a riding atom approximation with following constraints: $\mathrm{C}-\mathrm{H}=0.93,0.97 \AA$ and $\mathrm{N}-\mathrm{H}=0.86$ $\AA$, and with $U_{\text {iso }}(\mathrm{H})=1.2 U_{\mathrm{eq}}(\mathrm{C}$-aromatic and methylene and $N$-amido) in all the cases. In the absence of significant 
anomalous scattering effects, 1406 Friedel pairs were merged. The absolute configuration was determined by synthesis.

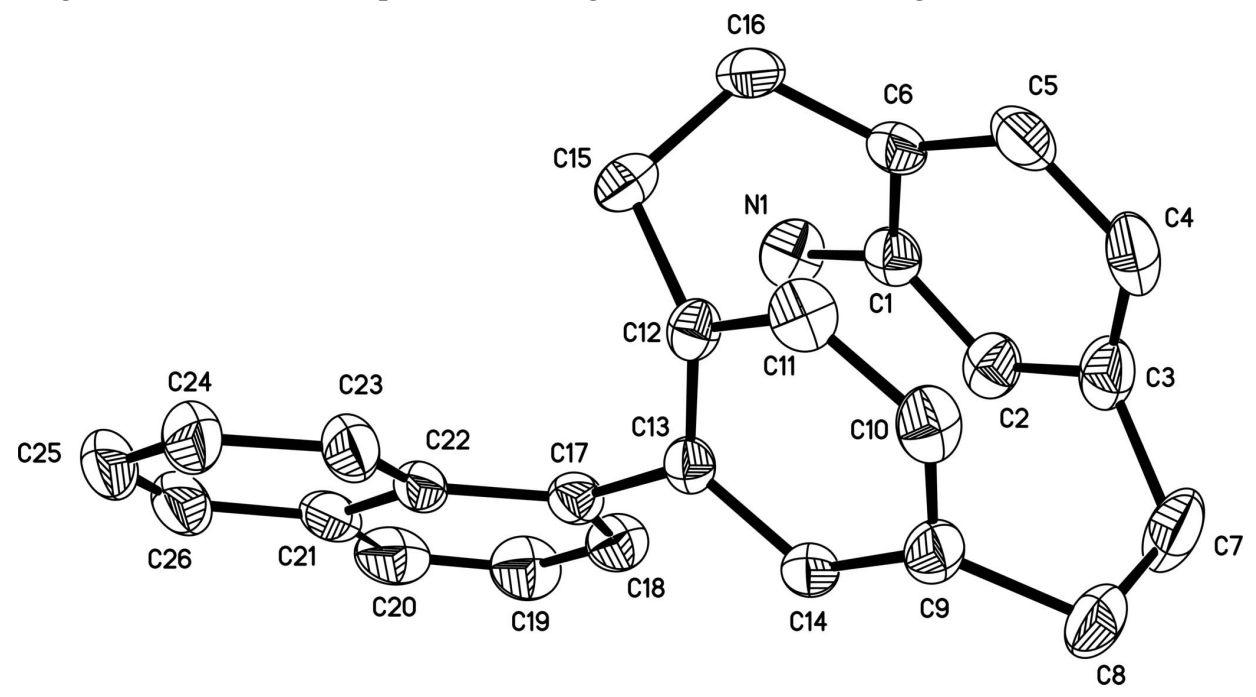

\section{Figure 1}

The structure of the title compound, showing $30 \%$ probability displacement ellipsoids and the atom-numbering scheme.

The $\mathrm{H}$ atoms are omitted.

\section{$1^{2}$-amino-4²-(1-naphthyl)-1,4(1,4)-dibenzenacyclohexaphane}

Crystal data

$\mathrm{C}_{26} \mathrm{H}_{23} \mathrm{~N}$

$M_{r}=349.45$

Orthorhombic, $P 2{ }_{1} 2_{1} 2_{1}$

$a=8.5261(5) \AA$

$b=12.8123(8) \AA$

$c=17.2065(11) \AA$

$V=1879.6(2) \AA^{3}$

$Z=4$

$F(000)=744$

\section{Data collection}

Bruker SMART APEXII CCD area-detector diffractometer

Radiation source: fine-focus sealed tube

Graphite monochromator

$\varphi$ and $\omega$ scans

Absorption correction: multi-scan

(SADABS; Sheldrick, 1996)

$T_{\min }=0.990, T_{\max }=0.993$

\section{Refinement}

Refinement on $F^{2}$

Least-squares matrix: full

$R\left[F^{2}>2 \sigma\left(F^{2}\right)\right]=0.054$

$w R\left(F^{2}\right)=0.159$

$S=1.00$

1901 reflections

245 parameters

6 restraints
$D_{\mathrm{x}}=1.235 \mathrm{Mg} \mathrm{m}^{-3}$

Mo $K \alpha$ radiation, $\lambda=0.71073 \AA$

Cell parameters from 3936 reflections

$\theta=2.7-25.1^{\circ}$

$\mu=0.07 \mathrm{~mm}^{-1}$

$T=293 \mathrm{~K}$

Block, colorless

$0.15 \times 0.12 \times 0.10 \mathrm{~mm}$

9879 measured reflections

1901 independent reflections

1690 reflections with $I>2 \sigma(I)$

$R_{\text {int }}=0.026$

$\theta_{\text {max }}=25.0^{\circ}, \theta_{\text {min }}=2.0^{\circ}$

$h=-8 \rightarrow 10$

$k=-14 \rightarrow 15$

$l=-12 \rightarrow 20$

Primary atom site location: structure-invariant direct methods

Secondary atom site location: difference Fourier map

Hydrogen site location: inferred from neighbouring sites

$\mathrm{H}$-atom parameters constrained 
$w=1 /\left[\sigma^{2}\left(F_{\mathrm{o}}^{2}\right)+(0.0986 P)^{2}+0.8216 P\right]$

where $P=\left(F_{\mathrm{o}}^{2}+2 F_{\mathrm{c}}{ }^{2}\right) / 3$

$(\Delta / \sigma)_{\max }=0.005$

$\Delta \rho_{\max }=0.37$ e $\AA^{-3}$
$\Delta \rho_{\min }=-0.33$ e $\AA^{-3}$

Extinction correction: SHELXL97 (Sheldrick, 2008), $\mathrm{Fc}^{*}=\mathrm{kFc}\left[1+0.001 \mathrm{xFc} \lambda^{3} / \sin (2 \theta)\right]^{-1 / 4}$

Extinction coefficient: $0.022(5)$

\section{Special details}

Geometry. All e.s.d.'s (except the e.s.d. in the dihedral angle between two l.s. planes) are estimated using the full covariance matrix. The cell e.s.d.'s are taken into account individually in the estimation of e.s.d.'s in distances, angles and torsion angles; correlations between e.s.d.'s in cell parameters are only used when they are defined by crystal symmetry. An approximate (isotropic) treatment of cell e.s.d.'s is used for estimating e.s.d.'s involving 1.s. planes.

Refinement. Refinement of $F^{2}$ against ALL reflections. The weighted $R$-factor $w R$ and goodness of fit $S$ are based on $F^{2}$, conventional $R$-factors $R$ are based on $F$, with $F$ set to zero for negative $F^{2}$. The threshold expression of $F^{2}>\sigma\left(F^{2}\right)$ is used only for calculating $R$-factors (gt) etc. and is not relevant to the choice of reflections for refinement. $R$-factors based on $F^{2}$ are statistically about twice as large as those based on $F$, and $R$ - factors based on ALL data will be even larger.

Fractional atomic coordinates and isotropic or equivalent isotropic displacement parameters $\left(\AA^{2}\right)$

\begin{tabular}{|c|c|c|c|c|}
\hline & $x$ & $y$ & $z$ & $U_{\text {iso }} * / U_{\text {eq }}$ \\
\hline N1 & $0.2691(6)$ & $0.4913(3)$ & $0.2196(3)$ & $0.0659(12)$ \\
\hline $\mathrm{H} 1 \mathrm{~A}$ & 0.2007 & 0.5066 & 0.2545 & $0.079^{*}$ \\
\hline H1B & 0.3499 & 0.5306 & 0.2131 & $0.079 *$ \\
\hline $\mathrm{C} 1$ & $0.2490(5)$ & $0.4028(3)$ & $0.1733(2)$ & 0.0497 (11) \\
\hline $\mathrm{C} 2$ & $0.1199(5)$ & $0.3383(4)$ & $0.1826(3)$ & $0.0561(12)$ \\
\hline $\mathrm{H} 2$ & 0.0486 & 0.3532 & 0.2219 & $0.067^{*}$ \\
\hline $\mathrm{C} 3$ & $0.0935(6)$ & $0.2543(4)$ & $0.1365(3)$ & $0.0628(13)$ \\
\hline $\mathrm{C} 4$ & $0.1940(7)$ & $0.2352(4)$ & $0.0744(3)$ & $0.0683(15)$ \\
\hline $\mathrm{H} 4$ & 0.1728 & 0.1820 & 0.0392 & $0.082 *$ \\
\hline $\mathrm{C} 5$ & $0.3267(7)$ & $0.2972(4)$ & $0.0662(3)$ & $0.0628(14)$ \\
\hline H5 & 0.3937 & 0.2856 & 0.0244 & $0.075^{*}$ \\
\hline C6 & $0.3618(6)$ & $0.3758(4)$ & 0.1187 (2) & $0.0515(11)$ \\
\hline $\mathrm{C} 7$ & $0.0003(7)$ & $0.1612(5)$ & 0.1707 (4) & $0.0833(19)$ \\
\hline H7A & -0.0545 & 0.1263 & 0.1287 & $0.100 *$ \\
\hline H7B & -0.0782 & 0.1882 & 0.2063 & $0.100^{*}$ \\
\hline $\mathrm{C} 8$ & $0.1025(7)$ & $0.0790(4)$ & $0.2147(4)$ & $0.0692(15)$ \\
\hline $\mathrm{H} 8 \mathrm{~A}$ & 0.0486 & 0.0596 & 0.2622 & $0.083^{*}$ \\
\hline H8B & 0.1106 & 0.0169 & 0.1827 & $0.083^{*}$ \\
\hline C9 & $0.2692(6)$ & $0.1156(3)$ & $0.2358(3)$ & 0.0517 (11) \\
\hline $\mathrm{C} 10$ & $0.3837(6)$ & $0.1093(3)$ & $0.1808(3)$ & $0.0539(12)$ \\
\hline $\mathrm{H} 10$ & 0.3753 & 0.0595 & 0.1417 & $0.065^{*}$ \\
\hline C11 & $0.5102(6)$ & $0.1742(3)$ & $0.1817(3)$ & 0.0515 (11) \\
\hline H11 & 0.5909 & 0.1640 & 0.1461 & $0.062 *$ \\
\hline $\mathrm{C} 12$ & $0.5201(5)$ & $0.2548(3)$ & $0.2347(2)$ & $0.0417(10)$ \\
\hline $\mathrm{C} 13$ & $0.4179(5)$ & $0.2553(3)$ & $0.2981(2)$ & $0.0394(9)$ \\
\hline C14 & $0.2894(5)$ & $0.1865(3)$ & $0.2960(2)$ & $0.0465(10)$ \\
\hline H14 & 0.2162 & 0.1885 & 0.3361 & $0.056^{*}$ \\
\hline $\mathrm{C} 15$ & $0.6056(5)$ & $0.3547(4)$ & $0.2093(3)$ & 0.0504 (11) \\
\hline H15A & 0.7152 & 0.3386 & 0.2001 & $0.061^{*}$ \\
\hline H15B & 0.6005 & 0.4055 & 0.2510 & $0.061 *$ \\
\hline C16 & $0.5336(6)$ & $0.4041(4)$ & $0.1335(3)$ & 0.0585 (13) \\
\hline
\end{tabular}




\begin{tabular}{lllll} 
H16A & 0.5423 & 0.4794 & 0.1370 & $0.070^{*}$ \\
H16B & 0.5955 & 0.3816 & 0.0892 & $0.070^{*}$ \\
C17 & $0.4316(5)$ & $0.3268(3)$ & $0.3660(2)$ & $0.0442(10)$ \\
C18 & $0.3029(6)$ & $0.3795(4)$ & $0.3928(3)$ & $0.0567(12)$ \\
H18 & 0.2071 & 0.3686 & 0.3683 & $0.068^{*}$ \\
C19 & $0.3088(8)$ & $0.4494(5)$ & $0.4558(3)$ & $0.0711(16)$ \\
H19 & 0.2190 & 0.4849 & 0.4713 & $0.085^{*}$ \\
C20 & $0.4445(9)$ & $0.4643(5)$ & $0.4931(3)$ & $0.0748(18)$ \\
H20 & 0.4484 & 0.5109 & 0.5345 & $0.090^{*}$ \\
C21 & $0.5830(7)$ & $0.4106(4)$ & $0.4706(3)$ & $0.0630(15)$ \\
C22 & $0.5777(6)$ & $0.3395(4)$ & $0.4069(2)$ & $0.0479(11)$ \\
C23 & $0.7156(6)$ & $0.2835(4)$ & $0.3886(3)$ & $0.0599(13)$ \\
H23 & 0.7143 & 0.2352 & 0.3482 & $0.072^{*}$ \\
C24 & $0.8502(7)$ & $0.2994(5)$ & $0.4293(4)$ & $0.0771(18)$ \\
H24 & 0.9397 & 0.2614 & 0.4170 & $0.093^{*}$ \\
C25 & $0.8549(10)$ & $0.3729(6)$ & $0.4899(4)$ & $0.091(2)$ \\
H25 & 0.9485 & 0.3850 & 0.5162 & $0.109^{*}$ \\
C26 & $0.7244(9)$ & $0.4258(5)$ & $0.5102(3)$ & $0.080(2)$ \\
H26 & 0.7285 & 0.4732 & 0.5512 & $0.096^{*}$ \\
& & & & \\
\hline
\end{tabular}

Atomic displacement parameters $\left(\AA^{2}\right)$

\begin{tabular}{lllllll}
\hline & $U^{11}$ & $U^{22}$ & $U^{33}$ & $U^{12}$ & $U^{13}$ & $U^{23}$ \\
\hline $\mathrm{N} 1$ & $0.069(3)$ & $0.048(2)$ & $0.081(3)$ & $0.005(2)$ & $-0.001(2)$ & $-0.009(2)$ \\
$\mathrm{C} 1$ & $0.055(3)$ & $0.042(2)$ & $0.052(2)$ & $0.007(2)$ & $-0.010(2)$ & $0.009(2)$ \\
$\mathrm{C} 2$ & $0.044(2)$ & $0.055(3)$ & $0.069(3)$ & $0.007(2)$ & $-0.011(2)$ & $0.005(2)$ \\
$\mathrm{C} 3$ & $0.054(3)$ & $0.062(3)$ & $0.073(3)$ & $-0.001(3)$ & $-0.022(3)$ & $0.002(3)$ \\
$\mathrm{C} 4$ & $0.084(4)$ & $0.060(3)$ & $0.061(3)$ & $-0.001(3)$ & $-0.033(3)$ & $-0.006(3)$ \\
$\mathrm{C} 5$ & $0.081(3)$ & $0.072(3)$ & $0.036(2)$ & $-0.002(3)$ & $-0.005(2)$ & $0.002(2)$ \\
$\mathrm{C} 6$ & $0.063(3)$ & $0.051(2)$ & $0.041(2)$ & $-0.006(2)$ & $-0.003(2)$ & $0.012(2)$ \\
$\mathrm{C} 7$ & $0.059(3)$ & $0.075(4)$ & $0.116(5)$ & $-0.024(3)$ & $-0.026(4)$ & $0.002(4)$ \\
$\mathrm{C} 8$ & $0.072(3)$ & $0.052(3)$ & $0.083(3)$ & $-0.026(3)$ & $-0.013(3)$ & $-0.002(3)$ \\
$\mathrm{C} 9$ & $0.062(3)$ & $0.035(2)$ & $0.058(2)$ & $-0.008(2)$ & $-0.011(2)$ & $0.0041(19)$ \\
$\mathrm{C} 10$ & $0.068(3)$ & $0.034(2)$ & $0.060(3)$ & $0.005(2)$ & $-0.011(2)$ & $-0.009(2)$ \\
$\mathrm{C} 11$ & $0.055(3)$ & $0.044(2)$ & $0.055(2)$ & $0.011(2)$ & $0.000(2)$ & $-0.007(2)$ \\
$\mathrm{C} 12$ & $0.0367(19)$ & $0.039(2)$ & $0.049(2)$ & $0.0018(18)$ & $-0.0046(18)$ & $-0.0019(18)$ \\
$\mathrm{C} 13$ & $0.043(2)$ & $0.0339(19)$ & $0.042(2)$ & $0.0011(17)$ & $-0.0071(17)$ & $0.0002(16)$ \\
$\mathrm{C} 14$ & $0.052(2)$ & $0.042(2)$ & $0.045(2)$ & $-0.006(2)$ & $-0.004(2)$ & $0.0073(19)$ \\
$\mathrm{C} 15$ & $0.042(2)$ & $0.049(2)$ & $0.060(3)$ & $-0.008(2)$ & $0.010(2)$ & $-0.003(2)$ \\
$\mathrm{C} 16$ & $0.065(3)$ & $0.062(3)$ & $0.049(2)$ & $-0.016(3)$ & $0.013(2)$ & $0.006(2)$ \\
$\mathrm{C} 17$ & $0.054(2)$ & $0.039(2)$ & $0.040(2)$ & $-0.0040(19)$ & $-0.001(2)$ & $0.0009(18)$ \\
$\mathrm{C} 18$ & $0.063(3)$ & $0.055(3)$ & $0.052(2)$ & $-0.003(2)$ & $0.009(2)$ & $-0.007(2)$ \\
$\mathrm{C} 19$ & $0.087(4)$ & $0.066(3)$ & $0.060(3)$ & $0.004(3)$ & $0.015(3)$ & $-0.014(3)$ \\
$\mathrm{C} 20$ & $0.117(5)$ & $0.060(3)$ & $0.047(3)$ & $-0.017(3)$ & $0.016(3)$ & $-0.013(2)$ \\
$\mathrm{C} 21$ & $0.096(4)$ & $0.055(3)$ & $0.038(2)$ & $-0.028(3)$ & $-0.010(3)$ & $0.009(2)$ \\
$\mathrm{C} 22$ & $0.061(3)$ & $0.043(2)$ & $0.040(2)$ & $-0.013(2)$ & $-0.009(2)$ & $0.0080(18)$ \\
$\mathrm{C} 23$ & $0.061(3)$ & $0.061(3)$ & $0.057(3)$ & $-0.004(2)$ & $-0.017(2)$ & $0.009(2)$ \\
$\mathrm{C} 24$ & $0.062(3)$ & $0.085(4)$ & $0.084(4)$ & $-0.012(3)$ & $-0.026(3)$ & $0.029(3)$ \\
& & & & &
\end{tabular}




$\begin{array}{lllllll}\mathrm{C} 25 & 0.101(5) & 0.105(5) & 0.068(4) & -0.048(5) & -0.044(4) & 0.026(4) \\ \mathrm{C} 26 & 0.104(5) & 0.083(4) & 0.054(3) & -0.035(4) & -0.028(3) & 0.013(3)\end{array}$

Geometric parameters $\left(\AA,{ }^{o}\right)$

\begin{tabular}{|c|c|c|c|}
\hline $\mathrm{N} 1-\mathrm{C} 1$ & $1.396(6)$ & $\mathrm{C} 12-\mathrm{C} 15$ & $1.537(6)$ \\
\hline $\mathrm{N} 1-\mathrm{H} 1 \mathrm{~A}$ & 0.8600 & $\mathrm{C} 13-\mathrm{C} 14$ & $1.406(6)$ \\
\hline N1-H1B & 0.8600 & $\mathrm{C} 13-\mathrm{C} 17$ & $1.489(6)$ \\
\hline $\mathrm{C} 1-\mathrm{C} 2$ & $1.386(7)$ & $\mathrm{C} 14-\mathrm{H} 14$ & 0.9300 \\
\hline $\mathrm{C} 1-\mathrm{C} 6$ & $1.389(7)$ & $\mathrm{C} 15-\mathrm{C} 16$ & $1.574(7)$ \\
\hline $\mathrm{C} 2-\mathrm{C} 3$ & $1.354(7)$ & C15-H15A & 0.9700 \\
\hline $\mathrm{C} 2-\mathrm{H} 2$ & 0.9300 & C15-H15B & 0.9700 \\
\hline $\mathrm{C} 3-\mathrm{C} 4$ & $1.391(8)$ & C16-H16A & 0.9700 \\
\hline $\mathrm{C} 3-\mathrm{C} 7$ & $1.549(8)$ & $\mathrm{C} 16-\mathrm{H} 16 \mathrm{~B}$ & 0.9700 \\
\hline $\mathrm{C} 4-\mathrm{C} 5$ & $1.390(8)$ & $\mathrm{C} 17-\mathrm{C} 18$ & $1.369(6)$ \\
\hline $\mathrm{C} 4-\mathrm{H} 4$ & 0.9300 & $\mathrm{C} 17-\mathrm{C} 22$ & $1.440(6)$ \\
\hline $\mathrm{C} 5-\mathrm{C} 6$ & $1.385(7)$ & $\mathrm{C} 18-\mathrm{C} 19$ & $1.406(7)$ \\
\hline $\mathrm{C} 5-\mathrm{H} 5$ & 0.9300 & $\mathrm{C} 18-\mathrm{H} 18$ & 0.9300 \\
\hline $\mathrm{C} 6-\mathrm{C} 16$ & $1.530(7)$ & $\mathrm{C} 19-\mathrm{C} 20$ & $1.337(9)$ \\
\hline $\mathrm{C} 7-\mathrm{C} 8$ & $1.563(8)$ & $\mathrm{C} 19-\mathrm{H} 19$ & 0.9300 \\
\hline C7-H7A & 0.9700 & $\mathrm{C} 20-\mathrm{C} 21$ & $1.421(9)$ \\
\hline C7-H7B & 0.9700 & $\mathrm{C} 20-\mathrm{H} 20$ & 0.9300 \\
\hline $\mathrm{C} 8-\mathrm{C} 9$ & $1.539(7)$ & $\mathrm{C} 21-\mathrm{C} 26$ & $1.398(8)$ \\
\hline $\mathrm{C} 8-\mathrm{H} 8 \mathrm{~A}$ & 0.9700 & $\mathrm{C} 21-\mathrm{C} 22$ & $1.426(7)$ \\
\hline $\mathrm{C} 8-\mathrm{H} 8 \mathrm{~B}$ & 0.9700 & $\mathrm{C} 22-\mathrm{C} 23$ & $1.413(7)$ \\
\hline $\mathrm{C} 9-\mathrm{C} 10$ & $1.362(7)$ & $\mathrm{C} 23-\mathrm{C} 24$ & $1.360(7)$ \\
\hline $\mathrm{C} 9-\mathrm{C} 14$ & $1.390(6)$ & $\mathrm{C} 23-\mathrm{H} 23$ & 0.9300 \\
\hline $\mathrm{C} 10-\mathrm{C} 11$ & $1.362(7)$ & $\mathrm{C} 24-\mathrm{C} 25$ & $1.405(10)$ \\
\hline $\mathrm{C} 10-\mathrm{H} 10$ & 0.9300 & $\mathrm{C} 24-\mathrm{H} 24$ & 0.9300 \\
\hline $\mathrm{C} 11-\mathrm{C} 12$ & $1.381(6)$ & $\mathrm{C} 25-\mathrm{C} 26$ & $1.349(10)$ \\
\hline $\mathrm{C} 11-\mathrm{H} 11$ & 0.9300 & $\mathrm{C} 25-\mathrm{H} 25$ & 0.9300 \\
\hline $\mathrm{C} 12-\mathrm{C} 13$ & $1.395(6)$ & $\mathrm{C} 26-\mathrm{H} 26$ & 0.9300 \\
\hline $\mathrm{C} 1-\mathrm{N} 1-\mathrm{H} 1 \mathrm{~A}$ & 120.0 & $\mathrm{C} 14-\mathrm{C} 13-\mathrm{C} 17$ & $117.8(4)$ \\
\hline $\mathrm{C} 1-\mathrm{N} 1-\mathrm{H} 1 \mathrm{~B}$ & 120.0 & $\mathrm{C} 9-\mathrm{C} 14-\mathrm{C} 13$ & $121.7(4)$ \\
\hline $\mathrm{H} 1 \mathrm{~A}-\mathrm{N} 1-\mathrm{H} 1 \mathrm{~B}$ & 120.0 & $\mathrm{C} 9-\mathrm{C} 14-\mathrm{H} 14$ & 119.2 \\
\hline $\mathrm{C} 2-\mathrm{C} 1-\mathrm{C} 6$ & $118.7(4)$ & $\mathrm{C} 13-\mathrm{C} 14-\mathrm{H} 14$ & 119.2 \\
\hline $\mathrm{C} 2-\mathrm{C} 1-\mathrm{N} 1$ & $121.1(5)$ & $\mathrm{C} 12-\mathrm{C} 15-\mathrm{C} 16$ & $112.7(4)$ \\
\hline $\mathrm{C} 6-\mathrm{C} 1-\mathrm{N} 1$ & $120.2(4)$ & $\mathrm{C} 12-\mathrm{C} 15-\mathrm{H} 15 \mathrm{~A}$ & 109.0 \\
\hline $\mathrm{C} 3-\mathrm{C} 2-\mathrm{C} 1$ & $122.6(5)$ & $\mathrm{C} 16-\mathrm{C} 15-\mathrm{H} 15 \mathrm{~A}$ & 109.0 \\
\hline $\mathrm{C} 3-\mathrm{C} 2-\mathrm{H} 2$ & 118.7 & $\mathrm{C} 12-\mathrm{C} 15-\mathrm{H} 15 \mathrm{~B}$ & 109.0 \\
\hline $\mathrm{C} 1-\mathrm{C} 2-\mathrm{H} 2$ & 118.7 & $\mathrm{C} 16-\mathrm{C} 15-\mathrm{H} 15 \mathrm{~B}$ & 109.0 \\
\hline $\mathrm{C} 2-\mathrm{C} 3-\mathrm{C} 4$ & $119.1(5)$ & $\mathrm{H} 15 \mathrm{~A}-\mathrm{C} 15-\mathrm{H} 15 \mathrm{~B}$ & 107.8 \\
\hline $\mathrm{C} 2-\mathrm{C} 3-\mathrm{C} 7$ & $118.3(5)$ & $\mathrm{C} 6-\mathrm{C} 16-\mathrm{C} 15$ & $114.6(4)$ \\
\hline $\mathrm{C} 4-\mathrm{C} 3-\mathrm{C} 7$ & $118.1(5)$ & $\mathrm{C} 6-\mathrm{C} 16-\mathrm{H} 16 \mathrm{~A}$ & 108.6 \\
\hline $\mathrm{C} 5-\mathrm{C} 4-\mathrm{C} 3$ & $118.6(5)$ & $\mathrm{C} 15-\mathrm{C} 16-\mathrm{H} 16 \mathrm{~A}$ & 108.6 \\
\hline $\mathrm{C} 5-\mathrm{C} 4-\mathrm{H} 4$ & 120.7 & $\mathrm{C} 6-\mathrm{C} 16-\mathrm{H} 16 \mathrm{~B}$ & 108.6 \\
\hline $\mathrm{C} 3-\mathrm{C} 4-\mathrm{H} 4$ & 120.7 & $\mathrm{C} 15-\mathrm{C} 16-\mathrm{H} 16 \mathrm{~B}$ & 108.6 \\
\hline
\end{tabular}




\begin{tabular}{|c|c|c|c|}
\hline $\mathrm{C} 6-\mathrm{C} 5-\mathrm{C} 4$ & $121.7(5)$ & $\mathrm{H} 16 \mathrm{~A}-\mathrm{C} 16-\mathrm{H} 16 \mathrm{~B}$ & 107.6 \\
\hline $\mathrm{C} 6-\mathrm{C} 5-\mathrm{H} 5$ & 119.2 & $\mathrm{C} 18-\mathrm{C} 17-\mathrm{C} 22$ & $118.2(4)$ \\
\hline $\mathrm{C} 4-\mathrm{C} 5-\mathrm{H} 5$ & 119.2 & $\mathrm{C} 18-\mathrm{C} 17-\mathrm{C} 13$ & $120.4(4)$ \\
\hline $\mathrm{C} 5-\mathrm{C} 6-\mathrm{C} 1$ & $118.2(5)$ & $\mathrm{C} 22-\mathrm{C} 17-\mathrm{C} 13$ & $121.4(4)$ \\
\hline $\mathrm{C} 5-\mathrm{C} 6-\mathrm{C} 16$ & $119.2(5)$ & $\mathrm{C} 17-\mathrm{C} 18-\mathrm{C} 19$ & $123.1(5)$ \\
\hline $\mathrm{C} 1-\mathrm{C} 6-\mathrm{C} 16$ & $119.4(4)$ & $\mathrm{C} 17-\mathrm{C} 18-\mathrm{H} 18$ & 118.5 \\
\hline $\mathrm{C} 3-\mathrm{C} 7-\mathrm{C} 8$ & $114.6(4)$ & $\mathrm{C} 19-\mathrm{C} 18-\mathrm{H} 18$ & 118.5 \\
\hline $\mathrm{C} 3-\mathrm{C} 7-\mathrm{H} 7 \mathrm{~A}$ & 108.6 & $\mathrm{C} 20-\mathrm{C} 19-\mathrm{C} 18$ & $119.4(6)$ \\
\hline $\mathrm{C} 8-\mathrm{C} 7-\mathrm{H} 7 \mathrm{~A}$ & 108.6 & $\mathrm{C} 20-\mathrm{C} 19-\mathrm{H} 19$ & 120.3 \\
\hline $\mathrm{C} 3-\mathrm{C} 7-\mathrm{H} 7 \mathrm{~B}$ & 108.6 & $\mathrm{C} 18-\mathrm{C} 19-\mathrm{H} 19$ & 120.3 \\
\hline $\mathrm{C} 8-\mathrm{C} 7-\mathrm{H} 7 \mathrm{~B}$ & 108.6 & $\mathrm{C} 19-\mathrm{C} 20-\mathrm{C} 21$ & $121.3(5)$ \\
\hline $\mathrm{H} 7 \mathrm{~A}-\mathrm{C} 7-\mathrm{H} 7 \mathrm{~B}$ & 107.6 & $\mathrm{C} 19-\mathrm{C} 20-\mathrm{H} 20$ & 119.3 \\
\hline $\mathrm{C} 9-\mathrm{C} 8-\mathrm{C} 7$ & $115.1(4)$ & $\mathrm{C} 21-\mathrm{C} 20-\mathrm{H} 20$ & 119.3 \\
\hline $\mathrm{C} 9-\mathrm{C} 8-\mathrm{H} 8 \mathrm{~A}$ & 108.5 & $\mathrm{C} 26-\mathrm{C} 21-\mathrm{C} 20$ & $121.1(5)$ \\
\hline $\mathrm{C} 7-\mathrm{C} 8-\mathrm{H} 8 \mathrm{~A}$ & 108.5 & $\mathrm{C} 26-\mathrm{C} 21-\mathrm{C} 22$ & $119.5(6)$ \\
\hline $\mathrm{C} 9-\mathrm{C} 8-\mathrm{H} 8 \mathrm{~B}$ & 108.5 & $\mathrm{C} 20-\mathrm{C} 21-\mathrm{C} 22$ & $119.5(5)$ \\
\hline $\mathrm{C} 7-\mathrm{C} 8-\mathrm{H} 8 \mathrm{~B}$ & 108.5 & $\mathrm{C} 23-\mathrm{C} 22-\mathrm{C} 21$ & $118.0(4)$ \\
\hline $\mathrm{H} 8 \mathrm{~A}-\mathrm{C} 8-\mathrm{H} 8 \mathrm{~B}$ & 107.5 & $\mathrm{C} 23-\mathrm{C} 22-\mathrm{C} 17$ & $123.6(4)$ \\
\hline $\mathrm{C} 10-\mathrm{C} 9-\mathrm{C} 14$ & $117.9(4)$ & $\mathrm{C} 21-\mathrm{C} 22-\mathrm{C} 17$ & $118.4(5)$ \\
\hline $\mathrm{C} 10-\mathrm{C} 9-\mathrm{C} 8$ & $118.7(4)$ & $\mathrm{C} 24-\mathrm{C} 23-\mathrm{C} 22$ & $120.7(5)$ \\
\hline $\mathrm{C} 14-\mathrm{C} 9-\mathrm{C} 8$ & $119.3(5)$ & $\mathrm{C} 24-\mathrm{C} 23-\mathrm{H} 23$ & 119.6 \\
\hline $\mathrm{C} 11-\mathrm{C} 10-\mathrm{C} 9$ & $121.6(4)$ & $\mathrm{C} 22-\mathrm{C} 23-\mathrm{H} 23$ & 119.6 \\
\hline $\mathrm{C} 11-\mathrm{C} 10-\mathrm{H} 10$ & 119.2 & $\mathrm{C} 23-\mathrm{C} 24-\mathrm{C} 25$ & $120.5(7)$ \\
\hline C9-C10-H10 & 119.2 & $\mathrm{C} 23-\mathrm{C} 24-\mathrm{H} 24$ & 119.8 \\
\hline $\mathrm{C} 10-\mathrm{C} 11-\mathrm{C} 12$ & $120.8(4)$ & $\mathrm{C} 25-\mathrm{C} 24-\mathrm{H} 24$ & 119.8 \\
\hline $\mathrm{C} 10-\mathrm{C} 11-\mathrm{H} 11$ & 119.6 & $\mathrm{C} 26-\mathrm{C} 25-\mathrm{C} 24$ & $120.3(6)$ \\
\hline $\mathrm{C} 12-\mathrm{C} 11-\mathrm{H} 11$ & 119.6 & $\mathrm{C} 26-\mathrm{C} 25-\mathrm{H} 25$ & 119.8 \\
\hline $\mathrm{C} 11-\mathrm{C} 12-\mathrm{C} 13$ & $118.8(4)$ & $\mathrm{C} 24-\mathrm{C} 25-\mathrm{H} 25$ & 119.8 \\
\hline $\mathrm{C} 11-\mathrm{C} 12-\mathrm{C} 15$ & $117.6(4)$ & $\mathrm{C} 25-\mathrm{C} 26-\mathrm{C} 21$ & $120.9(6)$ \\
\hline $\mathrm{C} 13-\mathrm{C} 12-\mathrm{C} 15$ & $121.0(4)$ & $\mathrm{C} 25-\mathrm{C} 26-\mathrm{H} 26$ & 119.5 \\
\hline $\mathrm{C} 12-\mathrm{C} 13-\mathrm{C} 14$ & $117.7(4)$ & $\mathrm{C} 21-\mathrm{C} 26-\mathrm{H} 26$ & 119.5 \\
\hline $\mathrm{C} 12-\mathrm{C} 13-\mathrm{C} 17$ & $124.5(4)$ & & \\
\hline $\mathrm{C} 6-\mathrm{C} 1-\mathrm{C} 2-\mathrm{C} 3$ & $-5.0(3)$ & $\mathrm{C} 11-\mathrm{C} 12-\mathrm{C} 15-\mathrm{C} 16$ & $56.6(5)$ \\
\hline $\mathrm{N} 1-\mathrm{C} 1-\mathrm{C} 2-\mathrm{C} 3$ & $177.2(4)$ & $\mathrm{C} 13-\mathrm{C} 12-\mathrm{C} 15-\mathrm{C} 16$ & $-104.9(5)$ \\
\hline $\mathrm{C} 1-\mathrm{C} 2-\mathrm{C} 3-\mathrm{C} 4$ & $-4.0(3)$ & $\mathrm{C} 5-\mathrm{C} 6-\mathrm{C} 16-\mathrm{C} 15$ & $-99.3(5)$ \\
\hline $\mathrm{C} 1-\mathrm{C} 2-\mathrm{C} 3-\mathrm{C} 7$ & $152.0(4)$ & $\mathrm{C} 1-\mathrm{C} 6-\mathrm{C} 16-\mathrm{C} 15$ & $60.0(6)$ \\
\hline $\mathrm{C} 2-\mathrm{C} 3-\mathrm{C} 4-\mathrm{C} 5$ & $6.0(5)$ & $\mathrm{C} 12-\mathrm{C} 15-\mathrm{C} 16-\mathrm{C} 6$ & $24.3(6)$ \\
\hline $\mathrm{C} 7-\mathrm{C} 3-\mathrm{C} 4-\mathrm{C} 5$ & $-150.0(5)$ & $\mathrm{C} 12-\mathrm{C} 13-\mathrm{C} 17-\mathrm{C} 18$ & $132.4(4)$ \\
\hline $\mathrm{C} 3-\mathrm{C} 4-\mathrm{C} 5-\mathrm{C} 6$ & $1.0(6)$ & $\mathrm{C} 14-\mathrm{C} 13-\mathrm{C} 17-\mathrm{C} 18$ & $-44.7(5)$ \\
\hline $\mathrm{C} 4-\mathrm{C} 5-\mathrm{C} 6-\mathrm{C} 1$ & $-9.9(6)$ & $\mathrm{C} 12-\mathrm{C} 13-\mathrm{C} 17-\mathrm{C} 22$ & $-50.0(6)$ \\
\hline $\mathrm{C} 4-\mathrm{C} 5-\mathrm{C} 6-\mathrm{C} 16$ & $149.7(4)$ & $\mathrm{C} 14-\mathrm{C} 13-\mathrm{C} 17-\mathrm{C} 22$ & $132.9(4)$ \\
\hline $\mathrm{C} 2-\mathrm{C} 1-\mathrm{C} 6-\mathrm{C} 5$ & $11.8(5)$ & $\mathrm{C} 22-\mathrm{C} 17-\mathrm{C} 18-\mathrm{C} 19$ & $3.4(7)$ \\
\hline $\mathrm{N} 1-\mathrm{C} 1-\mathrm{C} 6-\mathrm{C} 5$ & $-170.4(4)$ & $\mathrm{C} 13-\mathrm{C} 17-\mathrm{C} 18-\mathrm{C} 19$ & $-178.9(4)$ \\
\hline $\mathrm{C} 2-\mathrm{C} 1-\mathrm{C} 6-\mathrm{C} 16$ & $-147.8(4)$ & $\mathrm{C} 17-\mathrm{C} 18-\mathrm{C} 19-\mathrm{C} 20$ & $-1.6(8)$ \\
\hline $\mathrm{N} 1-\mathrm{C} 1-\mathrm{C} 6-\mathrm{C} 16$ & $30.0(6)$ & $\mathrm{C} 18-\mathrm{C} 19-\mathrm{C} 20-\mathrm{C} 21$ & $-0.3(9)$ \\
\hline $\mathrm{C} 2-\mathrm{C} 3-\mathrm{C} 7-\mathrm{C} 8$ & $-87.2(6)$ & $\mathrm{C} 19-\mathrm{C} 20-\mathrm{C} 21-\mathrm{C} 26$ & $-179.5(5)$ \\
\hline $\mathrm{C} 4-\mathrm{C} 3-\mathrm{C} 7-\mathrm{C} 8$ & $69.0(7)$ & $\mathrm{C} 19-\mathrm{C} 20-\mathrm{C} 21-\mathrm{C} 22$ & $0.3(8)$ \\
\hline
\end{tabular}




$\begin{array}{ll}\mathrm{C} 3-\mathrm{C} 7-\mathrm{C} 8-\mathrm{C} 9 & 15.1(8) \\ \mathrm{C} 7-\mathrm{C} 8-\mathrm{C} 9-\mathrm{C} 10 & -82.7(6) \\ \mathrm{C} 7-\mathrm{C} 8-\mathrm{C} 9-\mathrm{C} 14 & 74.1(7) \\ \mathrm{C} 14-\mathrm{C} 9-\mathrm{C} 10-\mathrm{C} 11 & -3.8(3) \\ \mathrm{C} 8-\mathrm{C} 9-\mathrm{C} 10-\mathrm{C} 11 & 153.4(4) \\ \mathrm{C} 9-\mathrm{C} 10-\mathrm{C} 11-\mathrm{C} 12 & -5.6(3) \\ \mathrm{C} 10-\mathrm{C} 11-\mathrm{C} 12-\mathrm{C} 13 & 14.2(5) \\ \mathrm{C} 10-\mathrm{C} 11-\mathrm{C} 12-\mathrm{C} 15 & -147.7(3) \\ \mathrm{C} 11-\mathrm{C} 12-\mathrm{C} 13-\mathrm{C} 14 & -13.1(5) \\ \mathrm{C} 15-\mathrm{C} 12-\mathrm{C} 13-\mathrm{C} 14 & 148.2(4) \\ \mathrm{C} 11-\mathrm{C} 12-\mathrm{C} 13-\mathrm{C} 17 & 169.7(4) \\ \mathrm{C} 15-\mathrm{C} 12-\mathrm{C} 13-\mathrm{C} 17 & -29.0(6) \\ \mathrm{C} 10-\mathrm{C} 9-\mathrm{C} 14-\mathrm{C} 13 & 4.5(5) \\ \mathrm{C} 8-\mathrm{C} 9-\mathrm{C} 14-\mathrm{C} 13 & -152.5(4) \\ \mathrm{C} 12-\mathrm{C} 13-\mathrm{C} 14-\mathrm{C} 9 & 4.0(5) \\ \mathrm{C} 17-\mathrm{C} 13-\mathrm{C} 14-\mathrm{C} 9 & -178.7(4)\end{array}$

$2.8(7)$

$-176.9(5)$

$-178.7(4)$

$1.6(7)$

$175.1(4)$

$-2.6(7)$

$-3.3(6)$

$179.0(4)$

$-1.8(7)$

$179.8(5)$

$-0.7(8)$

$2.4(9)$

$-1.4(9)$

$178.5(5)$

$-1.3(8)$ 